ORIGINAL ARTICLE

\title{
The effect of a symptom related "gating policy" on ANCA requests in routine clinical practice
}

\author{
D Sinclair, M Saas, J M Stevens
}

J Clin Pathol 2004;57:131-134. doi: 10.1136/¡cp2003.8052

See end of article for authors' affiliations

.....................

Correspondence to: Dr D Sinclair, Department of Chemical Pathology, Queen Alexandra Hospital, Portsmouth PO6 3 LY, UK; david.sinclair@ porthosp.nhs.uk

Accepted for publication 6 June 2003
Background: Most positive antineutrophil cytoplasmic antibody (ANCA) results are associated with nonvasculitic conditions, and guidelines have been proposed for the judicious use of this test. The outcome of applying similar guidelines in a routine laboratory is reported.

Methods: All immunology requests (6500) over six months were selected, and those requesting ANCA were studied for the appropriateness of the clinical data supporting the request, the presence of ANCA in those samples tested, and the final diagnosis. Antibodies were detected by indirect immunofluorescence. Results: ANCA testing was requested in 287 samples. Application of a "gating policy", which refuses analysis on requests that are not supported by clinical data suggestive of systemic vasculitis, made clinicians more selective about the patients for whom they requested ANCA testing. The percentage of "appropriate" screens for systemic vasculitis was relatively high (212 of 287 requests: $72.5 \%$ ). Only one of the remainder, for whom ANCA testing was initially refused, developed an ANCA related systemic vasculitis in the two years after the study, but the delay in reporting her positive ANCA was only two days. Most of the samples tested were negative (155 of 212), but most (42 of 57) of the patients with positive ANCA results were found to have a systemic vasculitis.

Conclusions: A gating policy to select requests supported by clinical data suggestive of systemic vasculitis makes ANCA testing more clinically relevant and cost effective. Studies where guidelines can be proposed and their effects measured are important in the light of clinical governance and evidence based medicine.
A ntineutrophil cytoplasmic antibodies (ANCA) were first described in $1982,{ }^{1}$ and have been established as useful markers for Wegener's granulomatosis and other systemic vasculitides. There is now an enormous literature on ANCA, and it is clear from published work that ANCA are found in several different conditions other than the systemic vasculitides, ${ }^{2-5}$ but their presence does not help in diagnosis.

In 1995, Edgar and colleagues ${ }^{6}$ published an audit of ANCA in routine clinical practice and reviewed the clinical usefulness of positive ANCA results in 1996. ${ }^{7}$ They reviewed the medical records of 301 of 327 consecutive patients in whom they had detected the presence of ANCA. In particular, they looked at the clinical diagnoses in which this antibody was found. The most frequent diagnosis was rheumatoid arthritis, with connective tissue disorders accounting for $27.9 \%$ of all patients. They also noted the presence of ANCA in a wide variety of non-vasculitic conditions, and they regarded these as "false positives". To gather information of this kind, it was necessary for them to test for ANCA in all patients for whom a request was made. They split their patients into four groups, namely:

- Group 1: Wegener's granulomatosis, microscopic polyangiitis, polyarteritis, syndrome of systemic vasculitis, Henoch-Schonlein purpura, Beçhet's and Churg-Strauss syndromes, and leucocytoclastic vasculitis (total 81 patients; $26.9 \%$ ).

- Group 2: rheumatoid arthritis, systemic lupus erythematosus (SLE), scleroderma, mixed connective tissue disease, juvenile chronic arthritis, and undifferentiated connective tissue disorder (total 84 patients; $27.9 \%$ ).

- Group 3: ulcerative colitis, Crohn's disease, sclerosing cholangitis, and polymyalgia rheumatica (total 18 patients; $5.9 \%$ ).
- Group 4: a wide variety of conditions including malignancy, infection, ischaemic heart disease, chronic active hepatitis, deep vein thrombosis, sarcoidosis, etc (total 118 patients; $39.2 \%$ ).

Their approach for this study was clinician led, and not part of a "gating" policy within the laboratory, and thus reflected clinical practice at that time. Their data and that of other published works ${ }^{8}$ show that less than half of all ANCA detected in an unselected group of patients are associated with a systemic vasculitis.

Our study sets out to investigate the effect on ANCA testing of a "gating" policy that we use to select patients in whom a defined number of clinical conditions or signs are present according to the clinical data supplied by the requesting physician. This policy has been in place for several years and is based on the conditions in which the knowledge of the presence of ANCA is known to be relevant. We routinely perform ANCA testing on the patients described in table 1 and we would normally reject analysis on all others. An "evidence base" for this approach came in the form of an international consensus statement, which was published in 1999, ${ }^{9}$ and that outlined the clinical criteria that should be applied to ANCA testing. These comprised: glomerulonephritis; pulmonary haemorrhage, especially pulmonary-renal syndrome; cutaneous vasculitis, especially with systemic features; multiple lung nodules; chronic destructive disease of the upper airways, long standing sinusitis, or otitis; subglottic tracheal stenosis; mononeuritis multiplex or peripheral neuropathy; and retro-orbital mass. With a few

Abbreviations: ANA, antinuclear antibodies; ANCA, antineutrophil cytoplasmic antibodies; FITC, fluorescein isothiocyanate; MPO, myeloperoxidase; PR3, proteinase 3; SLE, systemic lupus erythematosus 


\begin{tabular}{l} 
Table 1 Criteria applied to antineutrophil \\
cytoplasmic antibody requests \\
\hline Positive selection criteria \\
\hline Acute or chronic renal failure \\
Polyarteritis nodosa \\
Wegener's granulomatosis (established or tentative \\
diagnosis) \\
Proteinuria/haematuria requests from renal unit \\
Churg-Strauss syndrome \\
Nephrotic syndrome \\
Chest $x$ ray or lung biopsy abnormality \\
Haemoptysis \\
Nasal septum perforation \\
Henoch-Schonlein purpura \\
\hline
\end{tabular}

exceptions, these are very similar to the criteria applied by us and are strongly biased towards the investigation of symptoms attributable to a systemic vasculitis. We would define requests based on these clinical data as "appropriate" and it is noticeable that our patient grouping, group 1 in Edgar's audit, ${ }^{6}$ and the patients described in the consensus document ${ }^{9}$ are very similar. However, to our knowledge only one group has published the effect that the application of these or similar clinical criteria has on either requesting behaviour or the usefulness of the ANCA test. ${ }^{10}$

"Less than half of all antineutrophil cytoplasmic antibodies detected in an unselected group of patients are actually associated with a systemic vasculitis"

\section{MATERIALS AND METHODS}

ANCA were detected using an indirect immunofluorescence assay with "in house" ethanol fixed neutrophil preparations and fluorescein isothiocyanate (FITC) conjugated antihuman IgG (The Binding Site UK, Birmingham, UK). Serum was diluted 1/10 in phosphate buffered saline ( $\mathrm{pH}$ 7.3).

Antinuclear antibodies (ANA) were detected using indirect immunofluorescence of rat liver tissue. Serum diluted 1/20 in phosphate buffered saline ( $\mathrm{pH} 7.3$ ) was incubated with rat liver tissue and ANA were detected using FITC conjugated antihuman IgG (The Binding Site UK) and an Olympus BX40 fluorescence microscope. If ANA interfered with the ANCA patterns, antibodies to myeloperoxidase (MPO) and proteinase 3 (PR3) were confirmed using a dot blot assay (Biodiagnostics, Upton on Severn, Worcestershire, UK).

All consecutive request forms (6500) received by our department during a six month period were examined (January to June 2000). Those requesting ANCA were reviewed for clinical data and ANCA results, and the final diagnosis was reviewed by a consultant renal physician (JS) by study of laboratory results, pathology biopsy reports and individual patient case notes. After two years, we reviewed all laboratory results on patients for whom requests for the investigation of ANCA were made to ensure that no ANCA related vasculitis had developed during that time.

\section{RESULTS}

Figure 1 shows the results of the 287 requests that were made for ANCA testing during the six month period studied. "Appropriate" requests (those supported by clinical data suggestive of a systemic vasculitis) for ANCA investigations were made in $72.5 \%$ (208 of 287) patients compared with the $26.9 \%$ in group 1 patients reported by Edgar et al. ${ }^{6}$

The 75 of 287 patients in whom we refused ANCA investigations had a wide range of requesting symptoms,

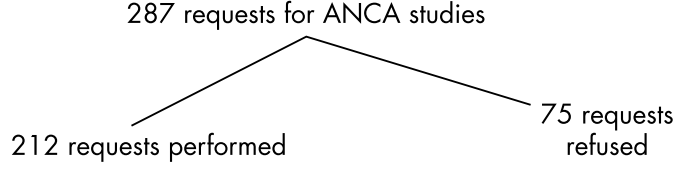

Clinical data

Numbers

ARF/CRF/ poor renal function $\quad 105$

Previous ANCA positive 38

Known Wegener's granulomatosis $\quad 37$

Nephrotic ?cause 23

?Wegener's granulomatosis 21

Chest $x$ ray abnormality $\quad 14$

Proteinuria/haematuria $\quad 13$

Churg-Strauss 7

Nasal septum perforation $\quad 5$

Polyarteritis nodosa 2

Leucocytoclastic vasculitis 1

Henoch-Schonlein pupura 1

* 1 each vasculitis/lupus; $\uparrow E S R$, myalgia 4

and night sweats; ?ulcerative colitis;

and anaemia ?cause

Figure 1 Clinical justification for antineutrophil cytoplasmic antibody (ANCA) requesting. *Requests made in person by hospital consultants. $\mathrm{ARF} / \mathrm{CRF}$, acute/chronic renal failure.

but the most common ones were increased erythrocyte sedimentation rate, anaemia, and joint pains, and also included clinical data like hypothyroidism, myeloma, primary biliary cirrhosis, and "some kind of weird vasculitis" (that is, predominantly group 4 in Edgar's classification). These were rejected and accompanied by a comment from one of us (DS) stating that the test did not appear to be supported by clinical data, but that we were willing to discuss the case further if necessary. Two years after the study was completed, we reviewed the pathology laboratory records of patients in whom we rejected the original ANCA request and found that in only one case was a diagnosis of systemic vasculitis subsequently made. This was a patient for whom the supporting clinical evidence for ANCA testing was "?mixed connective tissue disease". She went on to develop a rapidly progressive glomerulonephritis consistent with Wegener's granulomatosis. The time delay in performing ANCA studies was only two days, however, because a second sample was taken that was supported by the clinical data "episcleritis/ haematuria and proteinuria". This was tested for ANCA and a moderate degree of cytoplasmic ANCA positivity was detected.

ANCA assays were performed on a few occasions when the request was made in person by a consultant, even if the supporting clinical data fell outside our guidelines (four of 287).

Figure 2 shows the test outcomes and final diagnosis made on the patients in whom we tested for ANCA. Most of the samples tested were negative for ANCA (155 of 212). The patients with a positive ANCA pattern (57 of 212) consisted mainly of patients with known Wegener's granulomatosis or those in whom Wegener's was strongly suspected. Hence, most of these positive ANCA specimens have a cytoplasmic ANCA pattern. Most (42 of 57) of our positive ANCA samples were eventually found to be associated with an ANCA related vasculitis (34 Wegener's, two Goodpasture's, and five microscopic polyarteritis (now more commonly referred to as "microscopic polyangiitis"), and one Churg Strauss, with some patients being positive on more than one occasion). 
212 ANCA investigations carried out

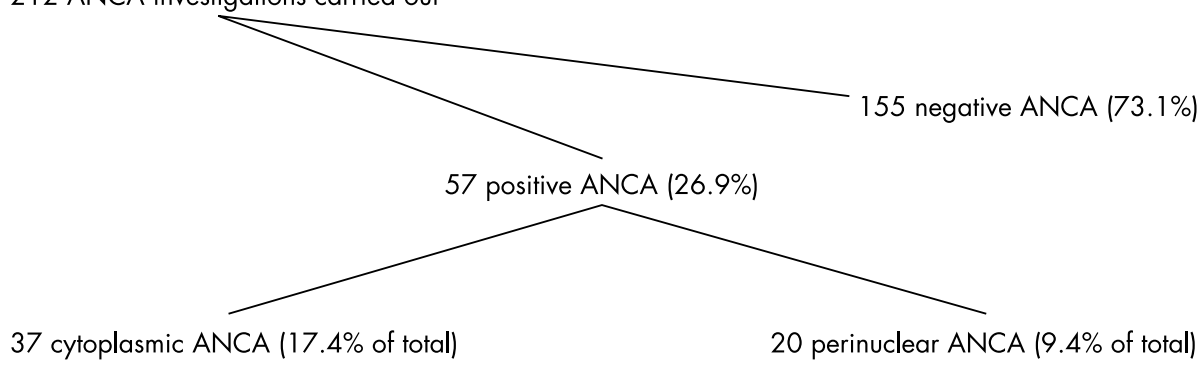

Figure 2 Outcome of the 57 patients who tested positive for ANCA. If ANA were present, then MPO/PR3 antibodies were confirmed using a dot blot technique. ANA, antinuclear antibodies; ANCA, antinuetrophil cytoplasmic antibodies; CAPD, continuous ambulatory peritoneal dialysis; ESRF, end stage renal failure; GBM, glomerular basement membrane; MPO, myeloperoxidase; PR3, proteinase 3; QC, quality control; SLE, systemic lupus erythematosis.

\author{
32 Wegener's or ?Wegener's \\ 1 ESRF on CAPD \\ 1 cystic fibrosis \\ 1 external QC sample \\ 1 anti-GBM antibody disease \\ 1 central retinal vein occlusion
}

\author{
5 microscopic polyangiitis \\ 2 anti-GBM antibody disease \\ 2 SLE cases (ANA negative) \\ 2 Wegener's granulomatosis \\ 1 ulcerative colitis \\ 1 pulmonary fibrosis \\ 1 renal transplant/glomerulonephritis \\ 1 deafness \\ 1 Churg-Strauss syndrome \\ 4 chronic renal failure
}

We calculated the number of ANCA tests performed as a proportion of the total number of routine autoimmunity tests (rheumatoid factor, antinuclear, antithyroid, anti-endomysium, antismooth muscle, antimitochondrial, anticardiolipin, and antidouble stranded DNA antibodies) performed between 1991 and 1992 and between 2002 and 2003. During the 1991-2 period, before the introduction of a gating system, ANCA tests represented 4.2\% (508 of 12 068) of the total workload of routine tests performed during that year. In 2002-3, ANCA requests had fallen to $2.4 \%$ (930 of 38543 ) of the tests performed. Our costs for ANCA testing are based on in house slides and amount to a modest $£ 2.91$ for each test. This represents a saving of approximately $£ 2004$ each year.

\section{DISCUSSION}

We have shown that our gating policy, which selects those requests supported by appropriate clinical data and rejects the more "speculative" test requests, has a positive impact on the usefulness of the test.

Our gating policy has been in place for 10 years and is biased towards the investigation of Wegener's granulomatosis and other systemic vasculitides, so a direct comparison with Edgar's data ${ }^{6}$ would not be possible because our laboratory has no "ungated" requests. However, assuming that the requesting clinicians described in Edgar's audit are unlikely to be very different from those involved in our study, this illustrates that the education process in place to guide clinicians in the most appropriate use of this test is having some effect. Most (73.4\%) of the requests we receive are appropriate, in that they are supported by clinical data suggestive of an ANCA related vasculitis. Some of the 151 of 212 patients who tested ANCA negative were patients with known ANCA related vasculitis, but who were in remission, or had a vasculitis diagnosed that would not normally be associated with ANCA. The presenting symptoms for such patients are similar to those presenting with an ANCA associated vasculitis, and as such they are still appropriate requests, although positive or negative predictive values for ANCA testing would not be relevant to this patient grouping.

If we compare our data in fig 2 with Edgar's unselected group, in which a minority of their ANCA positive results was associated with ANCA related vasculitis, it is clear that the gating policy adopted here has had an effect. This is not surprising, however, bearing in mind that the gating policy adopted by our laboratory is designed to identify such patients and exclude others.

We discourage the use of ANCA testing as a "screen for vasculitis", and demand evidence from our clinicians that a systemic vasculitis is more than something that they hope a negative ANCA will exclude. At the same time, these guidelines change with progress in defining the conditions-for example, we now include clinical signs such as scleritis, prolonged sinusitis, and sudden onset hearing loss in our current guidelines for "unquestioned ANCA testing". Edgar et al demonstrated in their audit that ANCA cannot confirm or exclude a diagnosis, but should only be used as part of a carefully considered investigative protocol. ${ }^{6}$ Their audit observed ANCA requesting in clinical practice and made recommendations as to its proper place. However, it was not possible for them to "close the loop" by observing the effects that changing requesting practices would have. Our study attempts to close that loop by demonstrating that restrictions placed on ANCA testing improve the cost and clinical effectiveness of the test by providing relevant information in patient groups in which the presence of ANCA may affect their care. McLaren et al looked at 2734 requests taken over a period of four years from 1996 to 2000 and showed that a large number of samples (88-100\%, depending on the department involved) that were ANCA positive had no "ANCA associated systemic vasculitis". They concluded that the test was being applied widely, but with very poor return, and their group proposed guidelines for more effective usage. ${ }^{11}$

The gold standard asked of any laboratory test by the requesting clinician is "Will the result alter my management?". Our data suggest that in some requests, at least, this standard may not be upheld at all times, because it is sometimes difficult to see what relevance the presence or absence of ANCA would have on some of the patients in whom the request was made. There are now several methods available for the detection and further identification of ANCA, but the method of choice for initial testing remains immunofluorescence using ethanol fixed neutrophil preparations. This is a labour intensive test and will add appreciable costs to any laboratory budget. In addition, the consensus documents published now recommend that all ANCA positive immunofluorescence results should be investigated further using a second technique to find out whether they are 


\section{Take home messages}

- The application of a "gating policy", which refuses requests that are not supported by clinical data suggestive of systemic vasculitis, makes clinicians more selective about the patients for whom they request antineutrophil cytoplasmic antibody (ANCA) testing

- In our laboratory, where a gating system is in place, the percentage of "appropriate" screens for systemic vasculitis was relatively high (212 of 287 requests: $72.5 \%)$

- A gating policy to select requests supported by clinical data suggestive of systemic vasculitis makes ANCA testing more clinically relevant and cost effective

myeloperoxidase or proteinase 3 positive. ${ }^{6}{ }^{911}$ We now comply with this, but this adds even more to the laboratory's costs, both in terms of consumables and staff time. If for no other reason than a financial one, it is important to direct this test towards the investigation of patients in whom there will be the maximum benefit.

"Restrictions placed on antineutrophil cytoplasmic antibody (ANCA) testing improve the cost and clinical effectiveness of the test by providing relevant information in patient groups in which the presence of ANCA may affect their care ${ }^{\prime \prime}$

Our laboratory serves a population in excess of 500000 people and a major renal medicine/transplant unit. The number of ANCA requests received appears to be much lower than might be expected for a laboratory and population of this size, and we suspect that this is a reflection of the success of the gating policy in educating clinicians and modifying requesting practices into a more patient focused approach.

However, the use of ANCA as a "screen" for vasculitis is an appealing one, in that it is tempting to draw parallels with the use of ANA as a screen for SLE. However, the incidence of ANA in SLE is high enough to conclude that a negative ANA does give useful information regarding the likelihood of SLE. We are not convinced that such a statement can be applied to ANCA in vasculitis-that is, a negative ANCA will not have the same significance regarding systemic vasculitis as a negative ANA does for SLE. However, some authors believe that a negative ANCA almost completely excludes an ANCA related vasculitis, although questioning its relevance in every positive case. ${ }^{12}$ We found a high incidence of ANCA negative microscopic polyarteritis/rapidly progressive glomerulonephritis in our series. ${ }^{13}$ Thus, we are left with a test whose unrestricted application gives most positive results in nonvasculitic conditions and for which negative results do not completely "exclude" systemic vasculitis. Mandl et al recently published an excellent retrospective study, ${ }^{10}$ in which they applied guidelines to existing ANCA requests and found that, had their guidelines been adhered to when the samples were received, ANCA testing would have fallen by $23 \%$ and the false positive rate for the detection of ANCA related vasculitis would have fallen by $27 \%$; however, most importantly, their study of case notes confirmed that no cases of ANCA associated vasculitis would have been missed by applying gating criteria. This paper effectively bears out the findings of our current study, but in a retrospective manner and using slightly different criteria, so a direct comparison is not possible. One of the main differences between the paper of Mandl et al and our current one is their inclusion of "cough" and "fever" as criteria to allow ANCA testing. We would reject analysis based on these two criteria if they were given as reasons for the request, with the proviso that discussion of each patient on an individual basis will allow ANCA testing to proceed.

We have itemised the cost savings that have resulted in our laboratory, but our figures do not include the extra expenditure required to investigate any gut related ANCA tests because we receive very few requests for these. Neither do they include the extra costs involved in the further investigation of any ANCA patterns not associated with ANCA related vasculitis (for example, all the ANCA patterns associated with Edgar's group 2, 3, and 4 patients would have to be analysed further for the presence of antibodies to MPO and PR3). Those laboratories performing ungated ANCA testing and/or using commercially available ANCA tests could realise considerable fiscal savings.

We have shown here that the implementation of investigative protocols based on published guidelines ${ }^{6-11}$ will have a major impact on the efficiency of ANCA testing, and large savings could be made by the implementation of such guidelines into routine clinical practice.

\section{Authors' affiliations}

D Sinclair, M Saas, Department of Chemical Pathology, Queen Alexandra Hospital, Portsmouth PO6 3 LY, UK

J M Stevens, Department of Renal Medicine, Queen Alexandra Hospital, Portsmouth PO6 3 LY, UK

\section{REFERENCES}

1 Davies DJ, Moran JE, Niall JF, et al. Segmental necrotising glomerulonephritis with antineutrophil antibody: possible arbovirus aetiology? BMJ 1982;285:606.

2 Terjung B, Worman HJ, Herzog V, et al. Differentiation of antineutrophil nuclear antibodies in inflammatory bowel and autoimmune liver diseases from antineutrophil cytoplasmic antibodies ( $\mathrm{p}$-ANCA) using immunofluorescence microscopy. Clin Exp Immunol 2001;126:37-46.

3 De Rosa FG, Amoroso A, Teggi A, et al. Anti-neutrophil cytoplasmic antibodies in Echinococcus granulosus hydatid disease. Hum Immunol 2001;62:1122-6.

4 Manolova I, Dancheva M, Halacheva K. Antineutrophil cytoplasmic antibodies in patients with systemic lupus erythematosus: prevalence, antigen specificity, and clinical associations. Rheumatol Int 2001;20:197-204.

5 Hoffman MD. Pyoderma gangrenosum associated with c-ANCA (h-lamp-2). Int J Dermatol 2001;40:135-7

6 Edgar JDM, McMillan SA, Bruce IN, et al. An audit of ANCA in routine clinical practice. Postgrad Med J 1995;71:605-612.

7 Edgar JDM. The clinical utility of ANCA positivity. Ann Rheum Dis 1996;55c:494-6

8 Zauli D, Grassi A, Ballardini G, et al. Testing for antineutrophil cytoplasmic antibodies. Am J Clin Pathol 2000;113:445-6.

9 Savige J, Gillis D, Benson E, et al. International consensus statement on testing and reporting of antineutrophil cytoplasmic antibodies (ANCA). Am J Clin Pathol 1999; 111:507-13.

10 Mandl LA, Solomon DH, Smith EL, et al. Using antineutrophil cytoplasmic antibody testing to diagnose vasculitis: can test-ordering guidelines improve diagnostic accuracy? Arch Intern Med 2002;162:1509-14.

11 McLaren JS, Stimson RH, McRorie ER, et al. The diagnostic value of antineutrophil cytoplasmic antibody testing in a routine clinical setting. QJM 2001;94:615-21.

12 Segelmark M, Westman K, Wieslander J. How and why should we detect ANCA? Clin Exp Rheumatol 2000;18:629-35.

13 Hedger NA, Roderick P, Drey N, et al. Incidence and outcome of pauciimmune rapidly progressive glomerulonephritis (RPGN) in Wessex, UK- a ten year retrospective study. Nephrol Dial Transplant 2000;15:1593-9. 